breast cancer risk factors which are hardly modifiable, the identification of occupational or environmental risk factors may be a key to prevention. It has been suggested that exposure to organic solvents in the workplace may play a role in the etiology of breast cancer. However, most epidemiological studies on solvents in female breast cancer have reported inconsistent results.

Methods We examined the risk of breast cancer related to lifetime exposure to chlorinated, petroleum-based and oxygenated solvents in the CECILE study, a large population-based casecontrol study carried out in France (2005-2007). 1230 women with breast cancer and 1315 population controls were included. Data collected included sociodemographic characteristics, medical and family history, anthropometric measurements, reproductive history, lifestyle habits, and lifetime occupational history. All jobs were coded using the International Standard Classification of Occupations (ISCO 1968) and the Classification of Activities in the European Community (NACE 1991). Exposure to five chlorinated, five petroleum and five oxygenated solvents was assessed using job-exposure matrices, which assigned indices of exposure (probability, frequency and intensity) for each job. A Cumulative Exposure Score (CES) taking into account probability, frequency, intensity and duration of exposure was calculated for each subject. Odds ratio (ORs) and their 95\% confidence intervals (95\% $\mathrm{CI})$ were estimated using unconditional logistic regression adjusting for potential confounders. Analyses were stratified by the menopausal status.

Results Significantly increased ORs were found in premenopausal women with high probability of exposure to alcohols $(1.50 ; 1.05-2.14)$ and diethyl ether $(1.97 ; 1.00-3.88)$. Premenopausal women with the highest CES of oxygenated solvents had an OR of $1.70(1.09-2.64) \quad(O R=1.67 ; 1.10-2.52$ for alcohols, 1.80 ; $0.95-3.42$ for diethyl ether); the highest CES of chlorinated solvents was associated with an OR of 2.14 (0.86-5.30). In premenopausal women, a duration of exposure to oxygenated solvents $\geq 25$ years with a probability of exposure $\geq 80 \%$ significantly increased the risk of breast cancer $(\mathrm{OR}=2.62 ; 1.27-5.40$ for all solvents, $2.72 ; 1.30-5.70$ for alcohols, and 2.79; 0.67-11.6 for diethyl ether). No association was observed for oxygenated, petroleum-based or chlorinated solvents in postmenopausal women.

Conclusion These findings suggest a role of occupational exposure to oxygenated solvents, notably to alcohols and diethyl ether, in the occurrence of breast cancer in premenopausal women. Our results highlight the importance of timing and duration of exposure for the study of occupational exposure to solvents in breast cancer risk.

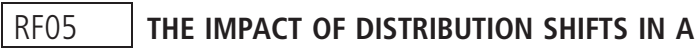 POPULATION'S CARDIOVASCULAR RISK FACTORS ON HEALTHY LIFE EXPECTANCY IN JAPAN}

\begin{abstract}
${ }^{1} \mathrm{R}$ Tsukinoki*, ${ }^{2} \mathrm{Y}$ Murakami, ${ }^{3,4} \mathrm{~K}$ Miura, ${ }^{5} \mathrm{~T}$ Okamura, ${ }^{3,4} \mathrm{~A}$ Kadota, ${ }^{6} \mathrm{~T}$ Hayakawa, ${ }^{7} \mathrm{~A}$ Okayama, ${ }^{3,4} \mathrm{H}$ Ueshima. 'Department of Community Health Nursing, apanese Red Cross College of Nursing, Tokyo, Japan; 'Department of Medical Statistics, School of Medicine, Toho University, Tokyo, Japan; ${ }^{3}$ Department of Public Health, Shiga University of Medical Sciences, Otsu, Japan; ${ }^{4}$ Center for Epidemiologic Research in Asia, Shiga University of Medical Sciences, Otsu, Japan; ${ }^{5}$ Department of Preventive Medicine and Public Health, Keio University, Tokyo, Japan; ${ }^{6}$ The Kinugasa Research Organization, Ritsumeikan University, Kyoto, Japan; ${ }^{7}$ Research Institute of Strategy for Prevention, Tokyo, Japan
\end{abstract}

10.1136/jech-2019-SSMabstracts. 120
Background Healthy life expectancy (HLE) is an important indicator of population health. Although the distribution of risk factors within populations has shifted during the past few decades (e.g., smoking rates have declined in developed countries), little is known if these changes affect population HLE. Our study aimed to estimate the impact of distribution shifts in a population's cardiovascular risk factors on HLE in Japan. Methods Data for analysis were obtained from NIPPON DATA90, a nationwide cohort study of over 9,000 Japanese people initiated in 1990. Using activities of daily living scores, we estimated the HLEs of study participants aged $\geq 60$ years at the baseline survey. We examined shifts in the following three risk factors: systolic blood pressure (SBP), body mass index (BMI), and smoking status. All HLE calculations were performed using maximum likelihood approach with interpolated Markov chains (iMaCh 0.98r7). First, risk factor-specific HLEs were calculated using multistate life tables. A total of 24 combinations of cardiovascular risk factors were estimated. Next, we calculated the population's baseline HLE (Scenario $0)$ using the weighted average of the risk factor-specific HLEs. The averaging weight was derived from the multivariable distribution of baseline data from NIPPON DATA90. Finally, scenarios were set to investigate the impact of distribution shifts in risk factors. In Scenario 1, the distributions of SBP and BMI were reduced by $4.0 \mathrm{mmHg}$ and $2.0 \mathrm{~kg} / \mathrm{m}^{2}$, respectively. In Scenario 2, we added the condition to Scenario 1 that all current smokers who wanted to quit smoking (men: 25\%, women: 40\%) were successful in quitting.

Results The analysis was performed using 6,676 participants (2,840 men and 3,836 women). The baseline HLE at age 60 years (Scenario 0) was 20.02 years in men and 24.32 years in women. After the shift in risk factors, the adjusted HLE at age 60 years in Scenario 1 was 20.12 years in men and 24.45 years in women, and the corresponding HLE in Scenario 2 was 20.46 years in men and 24.53 years in women. When compared with the baseline HLE, Scenario 1 presented a gain of 0.10 years in men and 0.14 years in women, and Scenario 2 presented a gain of 0.43 years in men and 0.21 years in women.

Conclusion We examined the impact of distribution shifts in SBP, BMI, and smoking status on population HLE in Japan, and found that smoking cessation substantially extended HLE.

\section{RF06 CHILDREN PRESENTING WITH CHRONIC PAIN TO THE ENGLISH NATIONAL HEALTH SERVICE: A WHOLE- POPULATION ADMINISTRATIVE DATA COHORT STUDY}

${ }^{1,2} \mathrm{MA}$ Jay*, ${ }^{2} \mathrm{RF}$ Howard, ${ }^{1} \mathrm{R}$ Gilbert. 'Population, Policy and Practice Programme, UCL GOS Institute of Child Health, London, UK; ' ${ }^{2}$ Department of Anaesthesia and Pain Medicine, Great Ormond Street Hospital for Children NHS Foundation Trust, London, UK

\subsection{6/jech-2019-SSMabstracts. 121}

Background Management of paediatric chronic pain (CP: pain $>3$ months' duration) is challenging and associated with significant comorbidities. Although large numbers of children may be affected, this population in England has not been well-described. In addition, despite partial provision of specialist NHS CP services, referral pathways are not well-established; children may therefore be receiving suboptimal interventions and access to $\mathrm{CP}$ services may be inequitable. We examined the characteristics and service utilisation of all children attending specialised CP services in England to inform commissioners and service providers. 